\title{
A Relação da Incidência de Casos de Dengue com a Precipitação na Área Urbana de Belém-PA, 2007 a 2011, Através de Modelos Multivariados de Séries Temporais
}

\author{
Ionara Santos Siqueira ${ }^{1}$ (iD, Joaquim Carlos Barbosa Queiroz ${ }^{1}$ (iD, Mario Miguel Amin $^{2}$ (iD, \\ Renata Kelen Cardoso Câmara ${ }^{1}$ (iD \\ ${ }^{1}$ Instituto de Geociências, Universidade Federal do Pará, Belém, PA, Brasil. \\ ${ }^{2}$ Núcleo de Altos Estudos Amazônicos, Universidade Federal do Pará, Belém, PA, Brasil.
}

Recebido em 15 de Março de 2015 - Aceito em 7 de Maio de 2018

\begin{abstract}
Resumo
Foi realizado um estudo de mapeamento de áreas de incidência e previsões para os casos de dengue na área urbana de Belém em que, para a realização de previsões de ocorrências de dengue, foi utilizada a Taxa de Incidência de Dengue (TID) relacionada à precipitação pluviométrica com uso de modelo multivariado baseado na metodologia de Box e Jenkins de séries temporais. O período do estudo foi de 5 anos (2007-2011). Os resultados das análises dos dados da TID e precipitação mostraram que, o aumento na TID é acompanhada por um aumento na precipitação, demonstrando uma relação direta entre essas variáveis no período de estudo, nos bairros da área urbana de Belém, dentre eles com maior destaque para os bairros Marco, Guamá e Pedreira. O modelo de previsão construído para a TID apresentou um bom ajuste com resultados satisfatórios podendo, neste caso, ser utilizado na previsão da dengue.
\end{abstract}

Palavras-chave: dengue, Belém, precipitação, modelos multivariados, séries temporais.

\section{The Relationship of the Incidence of Dengue Cases with the Precipitation in the Urban Area of Belém-PA, 2007 to 2011 Through Multivariate Models of Time Series}

\begin{abstract}
It was conducted a mapping study of the areas of incidence and predictions for dengue cases in the urban area of Belém, where the Dengue Incidence Rate (DIR) related to rainfall precipitation was used to forecast dengue occurrences using a multivariate model based on the Box and Jenkins time series methodology. The study period was 5 years (2007-2011). The results of the DIR data analysis and precipitation showed that the increase in DIR is accompanied by an increase in precipitation, demonstrating a direct relationship between these variables in the period under consideration, in the neighborhoods of the urban area of Belém, with greater emphasis in the neighborhoods of Marco, Guamá and Pedreira. The predictive model built for the DIR showed a good fit with satisfactory results and, in this case, can be used to predict dengue.
\end{abstract}

Keywords: dengue, Belém, precipitation, multivariate models, time series.

\section{Introdução}

Dengue é uma doença febril aguda transmitida aos humanos através de mosquito do gênero Aedes. Os países tropicais são os mais atingidos, devido suas características ambientais, climáticas e sociais. O clima é um importante fator na distribuição temporal das doenças transmitidas por vetores, como a dengue. Os estudos sobre as variáveis climáticas podem aperfeiçoar os conhecimentos sobre a sazonalidade e a predição de epidemias (Freitas et al., 2006), uma vez que a relação vetor-clima é tão importante quanto a relação vetor-homem.

A relação entre variáveis climáticas e a dengue tem sido investigada por diversos autores. Para tal, análise de séries temporais são frequentemente realizadas, com o in-

Autor de correspondência: Ionara Santos Siqueira, ionara_siqueira@yahoo.com.br. 
tuito de descrever a evolução temporal, identificar padrões e até mesmo predição (Hii et al., 2009); (Wu et al., 2007) (Liang et al., 2009); (Gasparrini e Armstrong, 2010).

Modelos matemáticos e estatísticos, mais especificamente as ferramentas de análises de séries temporais (Morettin, 2011), têm sido amplamente utilizados para monitorar e predizer a incidência de dengue e outras doenças infecciosas (ver por exemplo, Naing et al., 2002; Promprou et al., 2006; Wongkoon et al., 2007; Tian et al., 2008; Luz et al., 2008; Silawan et al., 2008; Choudhury et al., 2008; Soebiyanto et al., 2010; Wilder-Smith et al., 2010; Wongkoon et al., 2012; Earnest et al., 2012). Em populações específicas, estes modelos trouxeram contribuições sobre a compreensão da dinâmica da doença, permitindo predições do número de casos em períodos subsequentes à série estudada. Ao considerar variáveis climáticas (Hurtado-Díaz et al., 2007; Wu et al., 2007; Soebiyanto et al., 2010; Wongkoon et al., 2011; Gharbi et al., 2011), os modelos promovem entendimento do efeito sazonal da associação do clima sobre o aparecimento de casos da doença.

A dengue é uma doença infecciosa aguda, causada por um vírus da família flavivírus, de grande magnitude epidemiológica, ocasionando grandes epidemias no mundo principalmente nas regiões tropicais, atingindo milhões de pessoas anualmente (Barreto et al., 2008; Wilder-Smith et al., 2010).

Nas Américas, África, Ásia e Austrália, foram registradas pandemias e epidemias isoladas de dengue, principalmente nos três últimos séculos (Teixeira et al., 2005). Em 1981, ocorreu em Cuba, a primeira epidemia de dengue hemorrágica das Américas, quando foram registrados mais de 10.000 casos graves, relacionados ao sorotipo DEN-2. Entre 1981 e 1996, foram notificados 42.171 casos hemorrágicos em 25 países latino-americanos, sendo mais da metade procedentes de Cuba e Venezuela (Pinheiro, 1996; Kouri et al., 1998).

No Brasil, a primeira epidemia ocorreu em 1982, em Boa Vista, Roraima. Somente a partir de 1986, se iniciou como epidemias explosivas, que foram se expandindo para todas as regiões brasileiras (Causey e Theiler, 1958; Santos O.O, 2003).

Diversos trabalhos apontam que o período de maior risco de dengue no Brasil situa-se durante ou logo após a estação das chuvas (Natal, 2002; Ribeiro et al., 2006; Souza, 1999; Rebelo et al., 1999; Silva et al., 2006). Nestes períodos são observados picos epidêmicos nas cidades com colonização de Aedes aegypti e circulação viral, enquanto que nos demais meses do ano a incidência chega a números bastante reduzidos ou mesmo sem notificação.

Assim, a adoção de medidas que possam prever e diminuir a incidência de casos de dengue é um fator fundamental, para a aplicação de medidas mais eficazes por parte dos órgãos públicos, como sendo um incentivo para a redução de custos com a saúde, assim como em relação ao número de pessoas infectadas.

As previsões e o mapeamento de incidência de epidemias juntamente com medidas de controle podem contribuir para reduções significativas não somente em mortalidade, mas também em custos com a saúde. Neste sentido, o uso de modelos temporais para os casos de incidência de determinadas epidemias podem ser úteis para a previsão e controle de doenças. Portanto, o objetivo deste trabalho é analisar a incidência de casos de dengue com a precipitação, através de modelos multivariados de séries temporais para a realização de previsões de casos de dengue notificados na área urbana de Belém.

\section{Material e Métodos}

\section{1 Área de estudo}

De acordo com o Anuário Estatístico do Município de Belém - 2011, a área urbana do município possui coordenadas geográficas de $1^{\circ} 27^{\prime} 20^{\prime \prime}$ de Latitude $\mathrm{S}$ e de $48^{\circ} 30^{\prime} 15^{\prime \prime}$ de Longitude W. Sua população segundo estimativas do IBGE de 2010 é o equivalente a 1.392.031 habitantes, com uma área urbana formada por 71 bairros.

O clima da região é do tipo equatorial úmido, classificado segundo Koppen na categoria " $A$ ", com características gerais que permitem seu enquadramento nos subtipos "Af", "Aw" e "Am" (Cosanpa, 1987). A estação chuvosa desenvolve-se com maior intensidade de dezembro a junho e a estação de menor índice pluviométrico, que representa o "período seco", ocorre de julho a novembro. No entanto os meses não se apresentam bem definidos e não existe uma repartição muito uniforme das chuvas durante o ano (Sudam, 1984).

A Divisão Político-Administrativa de Belém está desmembrada em 08 Distritos Administrativos, compreendidos em Mosqueiro (DAMOS); Outeiro (DAOUT); Icoarací (DAICO); Benguí (DABEN); Entroncamento (DAENT); Sacramenta (DASAC); Belém (DABEL) e Guamá (DAGUA), Fig. 1.

\subsection{Casos de dengue}

No presente estudo são empregados casos mensais de dengue notificados à Secretaria Municipal de Saúde (SESMA), Departamento de Vigilância e Saúde (DEVS), no período de 2007 à 2011. Esses dados foram repassados através de um programa interno denominado de Sinan NET (Sistema de Informação de Agravos de Notificação), que vem a ser um sistema de informação nacional contínuo, universal e adequado à descentralização da vigilância, que engloba além da notificação, a investigação de agravos.

As taxas de incidência de dengue (TID) no período de 2007 à 2011 foram estimadas pela razão entre a soma de todos os casos notificados no período estudado por bairros e a soma da população do bairro, sendo o resultado multiplicado por 100000 . Foi construída uma série histórica para 


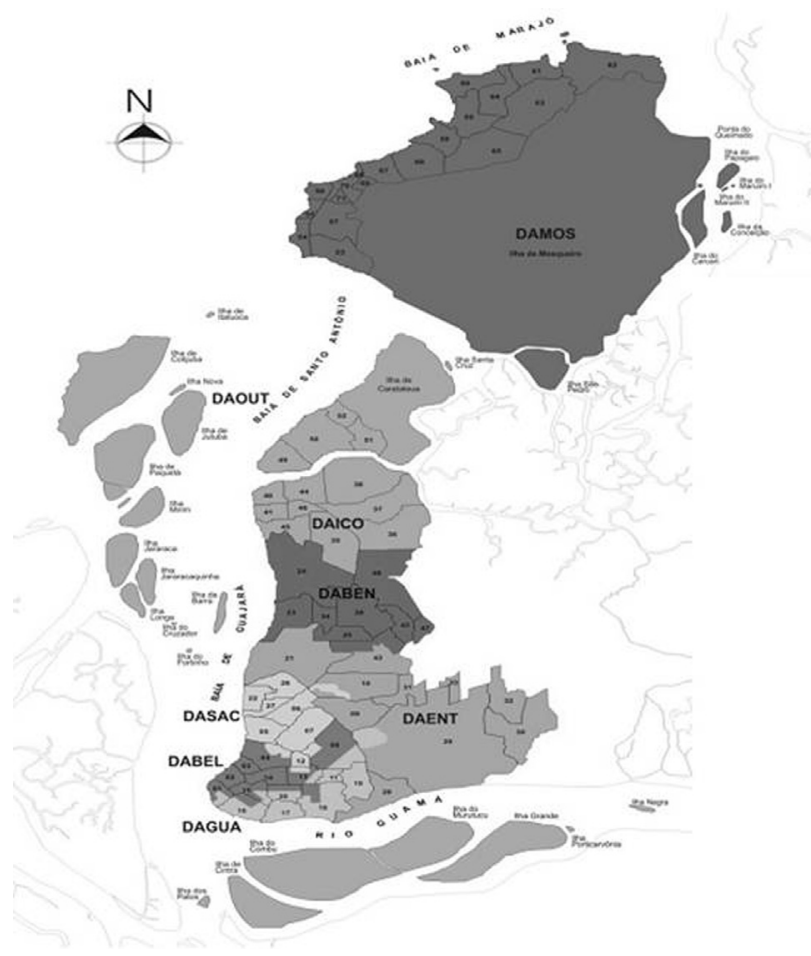

Figura 1 - Mapa de localização. Fonte: Anuário Estatístico do Município de Belém (2011).

mostrar a evolução da TID relacionada às precipitações médias mensais no período de estudo.

\subsection{Precipitação pluviométrica}

Foram utilizados dados totais mensais de precipitação pluviométrica em milímetro, como variável de entrada nos modelos multivariados. Esta variável climática foi selecionada para verificar a existência de correlação com a ocorrência do mosquito. Esses dados foram obtidos a partir do Banco de Dados fornecidos pelo Instituto Nacional de Meteorologia (INMET), no período compreendido entre 2007 à 2011. Para as análises dos casos de dengue e dados de precipitação para o período estudado foi utilizado o programa SAS.

\subsection{Análise de séries temporais}

Para a realização das previsões de casos de dengue em Belém, foi utilizada a metodologia de Box e Jenkins em modelos multivariados de séries temporais baseados em funções de transferência (Wei, 1990). Nesse caso, a Taxa de Incidência de Dengue (TID) foi considerada como variável resposta $\left(Y_{t}\right)$ e as precipitações como variável explicativa $\left(X_{t}\right)$.

Em um sistema linear de entrada e saída simples, as séries de entrada $\left(X_{t}\right)$ e saída $\left(Y_{t}\right)$ são relacionadas por meio de um filtro linear, como:

$$
Y_{t}=v(B) X_{t}+N_{t}
$$

onde $v(B)=\sum_{-\infty}^{\infty} v_{j} B^{j}$ refere-se a um filtro de função de transferência e $N_{t}$ a uma série de ruídos do sistema independente da série de entrada $\left(X_{t}\right)$.

\subsection{Validação do modelo}

A etapa final do processo de construção de modelos é a verificação de que o modelo experimental, dentro do seu domínio de aplicação, se comporta de maneira suficientemente satisfatória com relação aos objetivos do estudo. Esta etapa, chamada de validação do modelo, é o conjunto de condições prescritas para as quais o modelo experimental foi testado, comparado com o sistema real e julgado apto para uso.

Desse modo, dados não inclusos na modelagem da série temporal de casos ocorridos, servirão para permitir a validação do modelo, pela comparação dos valores estimados com os valores realmente observados, de modo que se possa avaliar a precisão das previsões.

Neste trabalho, para o desenvolvimento do modelo de séries temporais, foi utilizada a série de 60 observações compreendida entre os meses de Janeiro de 2007 a Dezembro de 2011, sendo que as 06 últimas observações, Julho a Dezembro de 2011, foram inseridas para comparar com as previsões obtidas pelo modelo e avaliar o desempenho do mesmo.

\section{Resultados e Discussão}

\subsection{Análises dos dados de ocorrência de casos de dengue (TID) e precipitação na área urbana de Belém}

Na Fig. 2 são apresentadas séries da Taxa de Incidência de Dengue TID e precipitação na área urbana de Belém. Observa-se que as séries não apresentam tendência, mas há indícios da presença de sazonalidade. Períodos em que o aumento no número de casos de dengue acompanha o aumento na quantidade de chuvas, demonstrando a relação entre o número de casos de dengue notificados e a precipitação nos anos de 2007 à 2011, evidenciando uma relação quando se inicia o período chuvoso (dezembro à maio) para cada ano considerado na pesquisa, observando que o mês de março de 2007 é o de maior evidencia, já com relação à precipitação a maior quantidade de chuvas é observado em março de 2009, mês este inserido no período chuvoso. No gráfico da Fig. 3 evidencia-se que os bairros do Marco, Guamá e Pedreira são os mais significativos, atingindo o total de 1.668 , 1.528 e 1.304 casos notificados respectivamente, período de 2007 à 2011, para esses bairros devemos considerar que os casos mais freqüentes estão concentrados em áreas com infraestrutura e também sem saneamento básico, pois o bairro que mostra bem isto é o bairro do Marco e Pedreira por serem bairros que apresentam áreas com infraestrutura e periferias. Áreas nobres possuem residências com piscinas, objetos ornamentais como vasos entre outros, o que torna criadouros potenciais, em contra- 
partida áreas de periferias possuem precárias condições de saneamento, maior acúmulo de lixo. Na Fig. 4 tem-se as precipitações médias mensais para o mesmo período.
O modelo univariado ajustado para a Taxa de Incidência de Dengue (TID) é mostrado pela Eq. (2), em que $B$ é o operador diferença, $B^{k} Y_{t}=Y_{-t-k}$ e nível descritivo, $p$, é

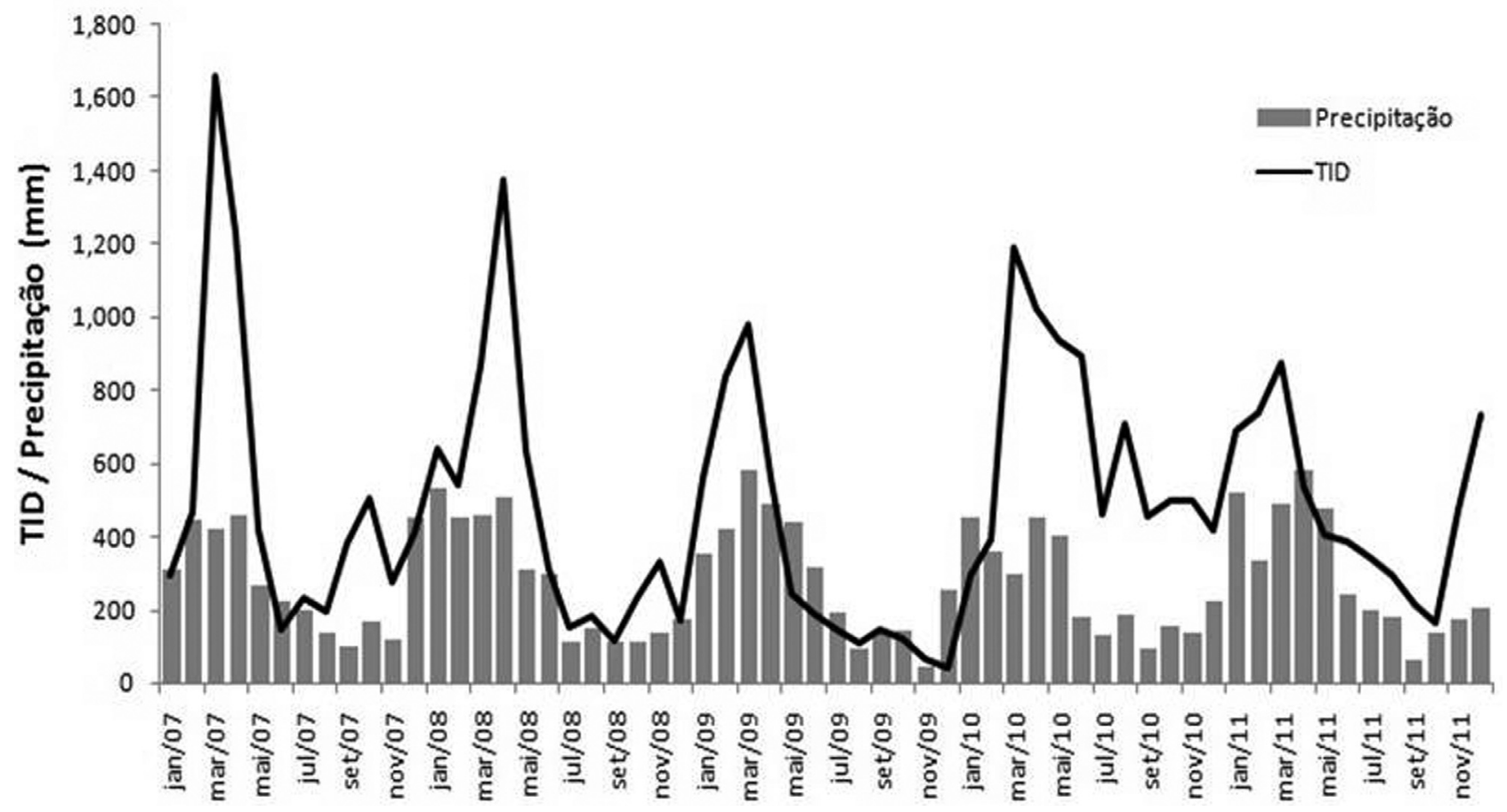

Figura 2 - Taxa de incidência de dengue e precipitação no período de 2007 a 2011.

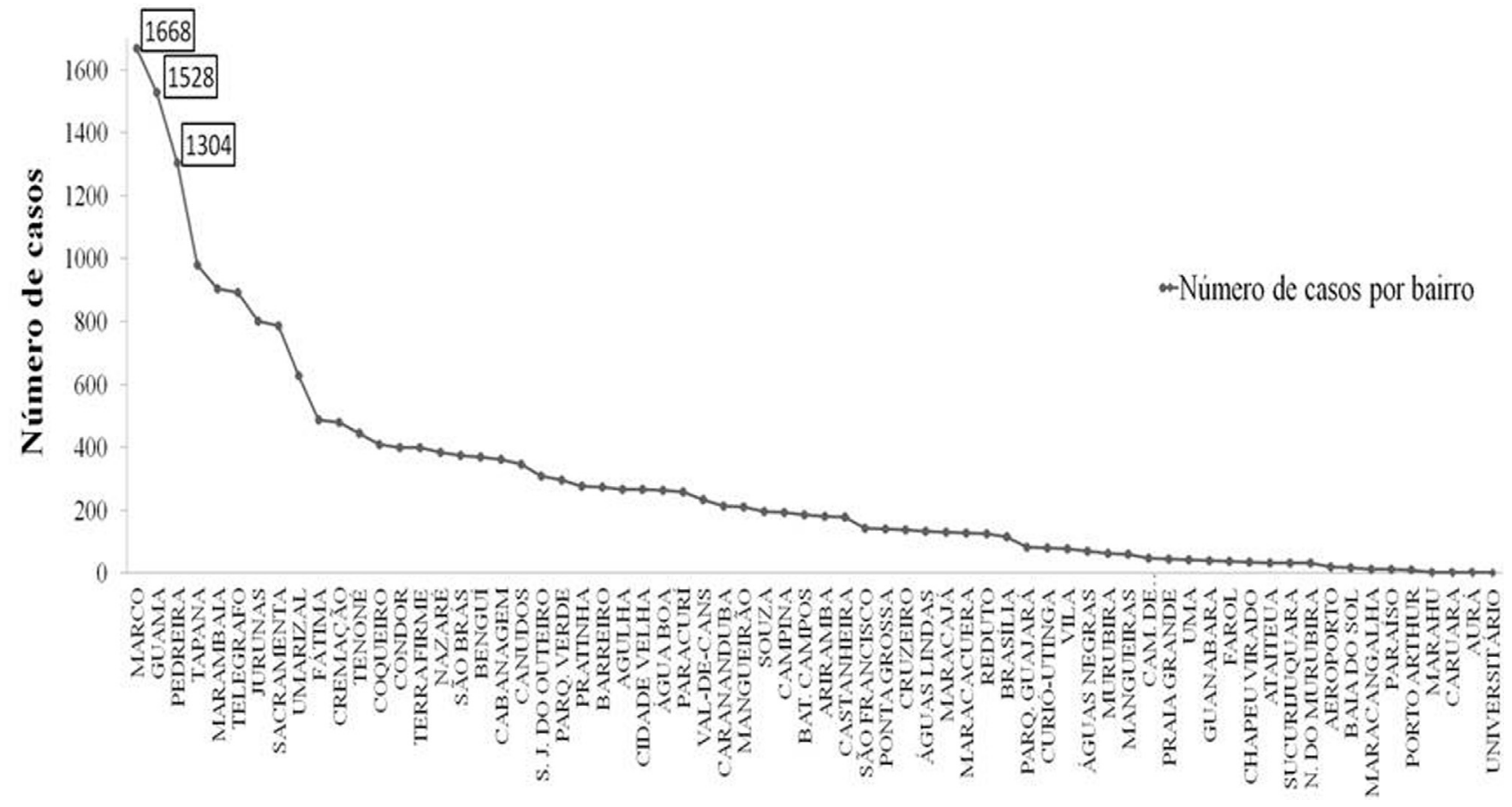

Figura 3 - Número de casos de dengue por bairros no período de 2007 a 2011. 


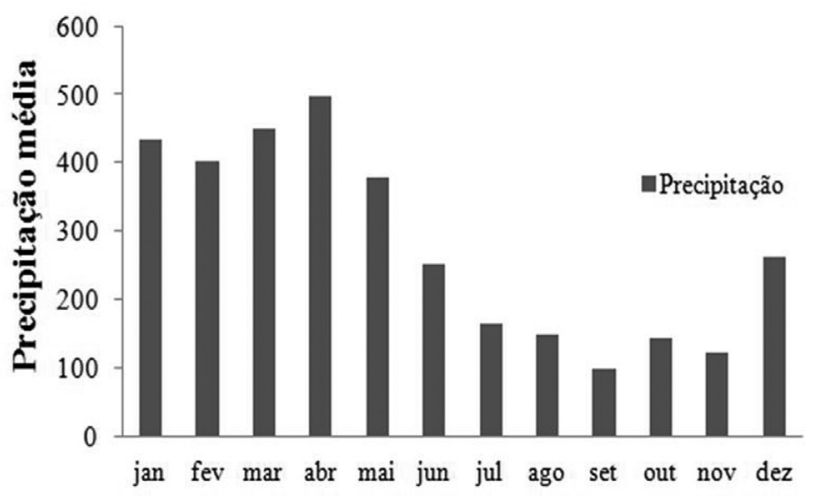

Figura 4 - Precipitação média mensal da área urbana de Belém no período de 2007 a 2011.

fornecido abaixo de cada estimativa dos parâmetros do modelo.

$$
\begin{aligned}
& \left.\underset{p<0,0001}{(1-0,678 B}+\underset{p<0,008}{0,373 B^{12}}\right) Y_{T}=\underset{p<0,0001}{486,93} \\
& +\underset{p<0,0033}{\left(1-0,439 B^{2}-\underset{p<0,0136}{0,347 B^{8}}\right) u_{t}}
\end{aligned}
$$

Na Fig. 5 é apresentado o gráfico do modelo univariado ajustado aos dados observados.

A Eq. (3) mostra o modelo univariado ajustado para a precipitação

$$
\left.\underset{p<0,0017}{(1-0,321 B}+\underset{p<0,0009}{0,346 B^{5}}+\underset{p<0,0159}{0,349 B^{6}}\right) Y_{T}=\underset{p<0,0001}{2280,26}+u_{t}
$$

Para o modelo multivariado, o filtro de pré-ajustamento para a série de entrada (precipitação) foi utilizado na série de saída Taxa de Incidência de Dengue (TID). Na Fig. 6 é apresentado o gráfico das correlações cruzadas entre a série resposta $Y_{t}$, Taxa de Incidência de Dengue (TID), e a série filtrada precipitação, assim como os resultados dessas correlações com a variável pré-ajustada. Observa-se um pico significativo no $\operatorname{lag} 0$, indicando que as séries estão em fase, ou seja, um aumento na precipitação é acompanhado ao mesmo tempo por um aumento nos casos de dengue.

O modelo ajustado para um intervalo de confiança de 95\% juntamente com os dados observados para a Taxa de Incidência de Dengue (TID) é apresentado na Eq. (4) e Fig. 7, evidenciando-se que o modelo ajustado representou bem o modelo previsto (abaixo de cada estimativa na Eq. (4), é apresentado o nível descritivo, $p$, significativo para todos os parâmetros estimados). Os resultados das estimativas do modelo e estatísticas de ajuste são:

$$
Y_{t}=\underset{p<0,0003}{\underset{p<0,0002}{1,055-0,466 B})} X_{t}+\frac{N_{t}}{\underset{\substack{(1-0,362 B) \\ p<0,045}}{p<0}}
$$

onde $Y_{t}$ representa a Taxa de Incidência de Dengue (TID) e $X_{t}$ a precipitação.

\subsection{Validação do modelo}

Para validação do modelo foram realizadas 6 previsões para a ocorrências de dengue, representadas pela Taxa de Incidência de Dengue (TID) para o periodo de julho a dezembro de 2011. Esse periodo não foi considerado na construção do modelo (Eq. (4)). As previsões foram então comparadas com as ocorrências observadas para a Taxa de Incidência de Dengue (TID). Os resultados são apresentadas na Tabela 1 e Fig. 8, onde se observa que as previsões apresentaram valores abaixo dos observados para a Taxa de Incidência de Dengue (TID). Entretanto, todas as previsões ficaram dentro do intervalo de confiança de $95 \%$ indicando

Dengue

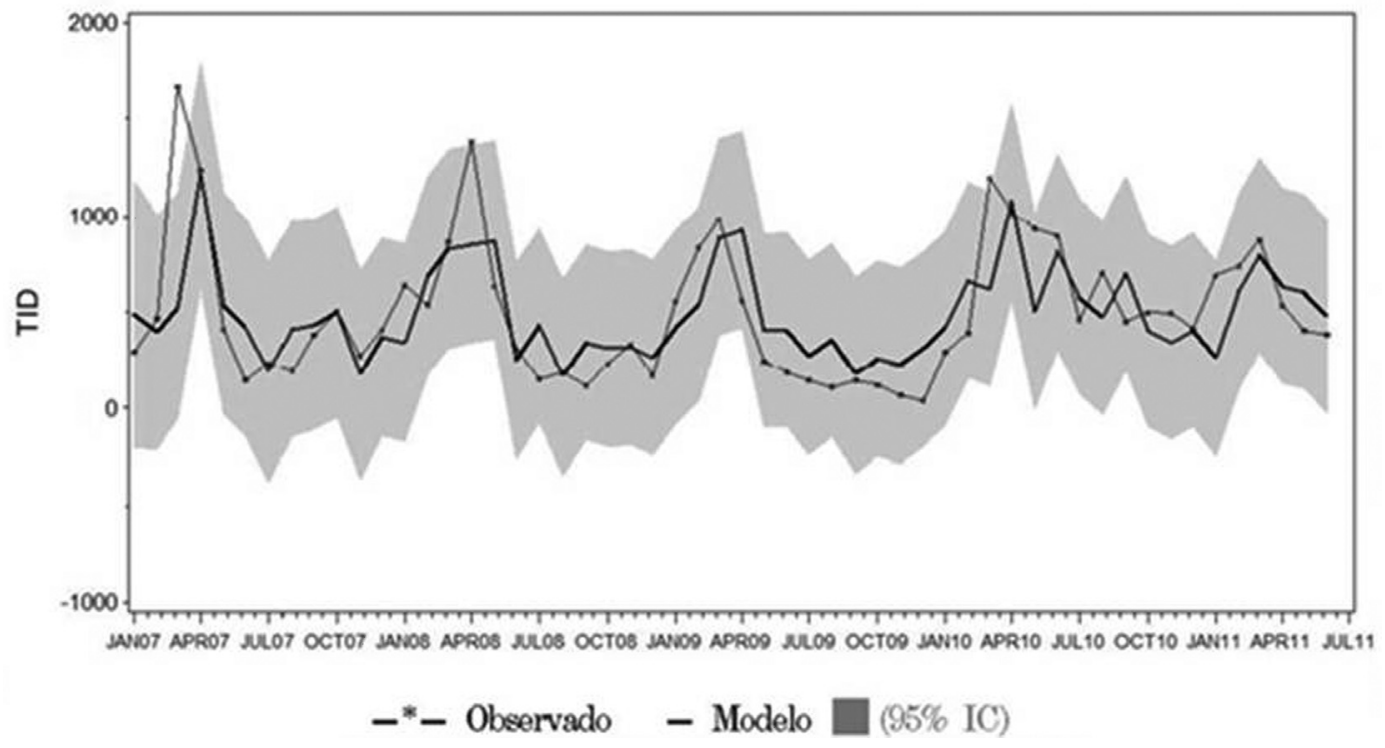

Figura 5 - Modelo univariado da TID ajustado. 
Correlacao Cruzada Tid vs Precip

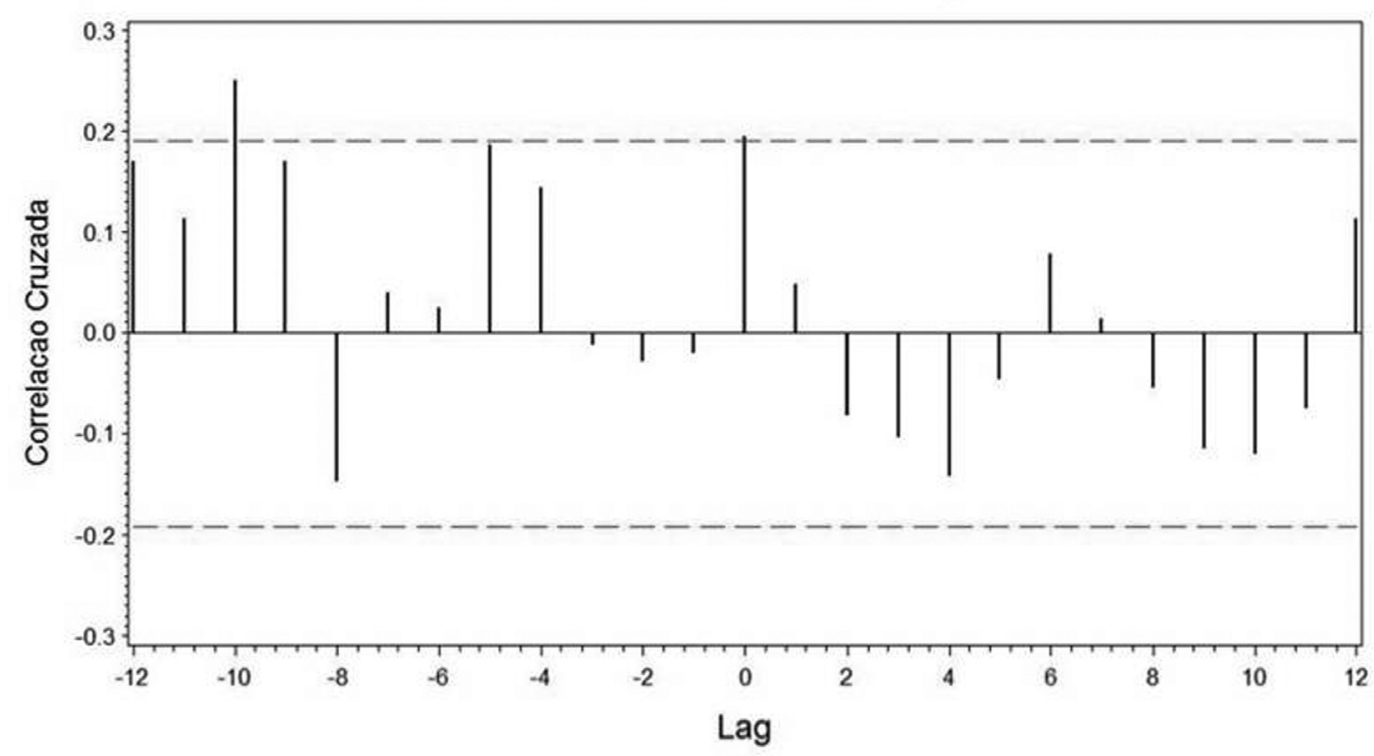

Figura 6 - Correlações cruzadas entre a TID e a precipitação.

Dengue

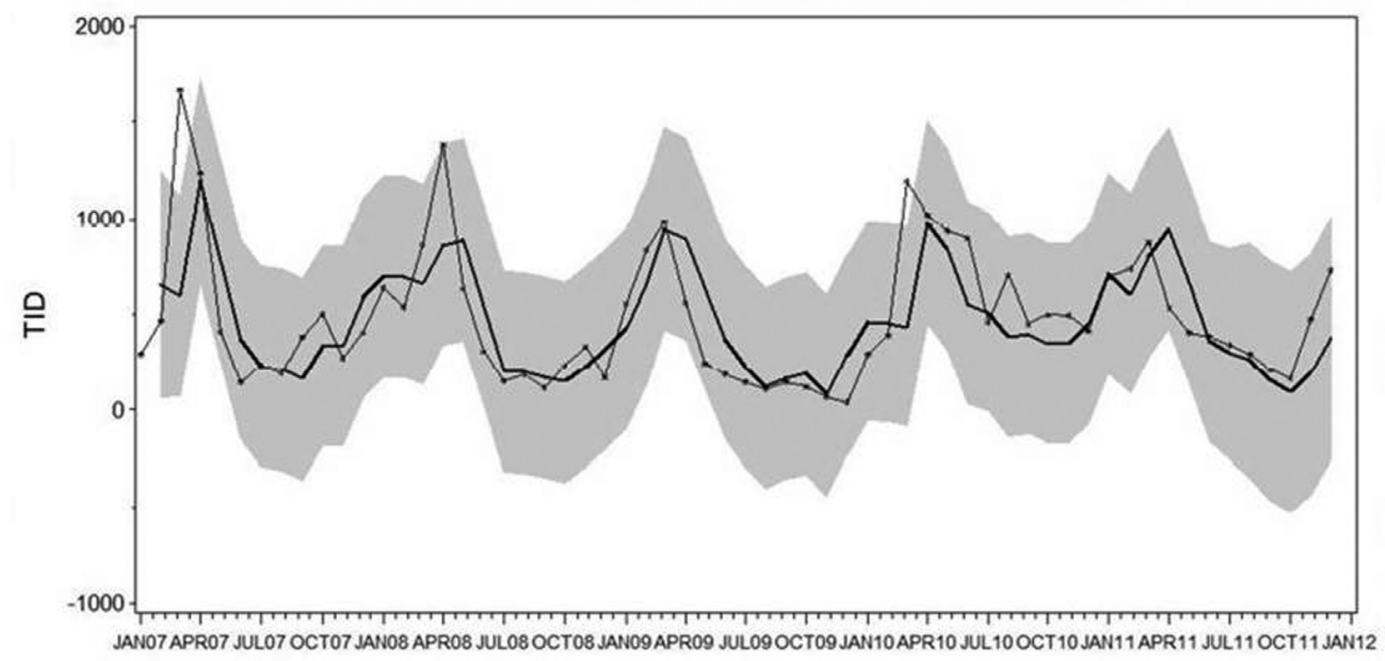

-*- Observado - Modelo $\square(95 \%$ IC)

Figura 7 - Modelo multivariado ajustado para a TID.

que as diferenças entre as previsões e valores observados são estatisticamente não significativos. Sabe-se porém, que amostras pequenas (menor do que em torno de 50 observações) produzem intervalos de confiança mais largos, isto é, com menor confiabilidade. Isso pode conduzir a conclusões equivocadas a respeito das significancias dos valores estimados (previstos). Como os intervalos de confiança para as previsões foram relativamente largos neste estudo, foi realizada uma análise estatística para uma melhor ava- liação das previsões da Taxa de Incidência de Dengue (TID) obtidas pelo modelo.

Inicialmente, foram calculados os erros médios entre os valores observados e os previstos em relação aos observados para a Taxa de Incidência de Dengue (TID) (Moretin, 2011) da Tabela 1. Os resultados foram 0,3383 para o Erro médio precentual absoluto (MAPE) e 0,3835 para a Raiz do erro quadrático médio (RMSE). Esses erros podem ser considerados relativamente altos, porém com o uso de uma 
TID (Previsao 6 meses)

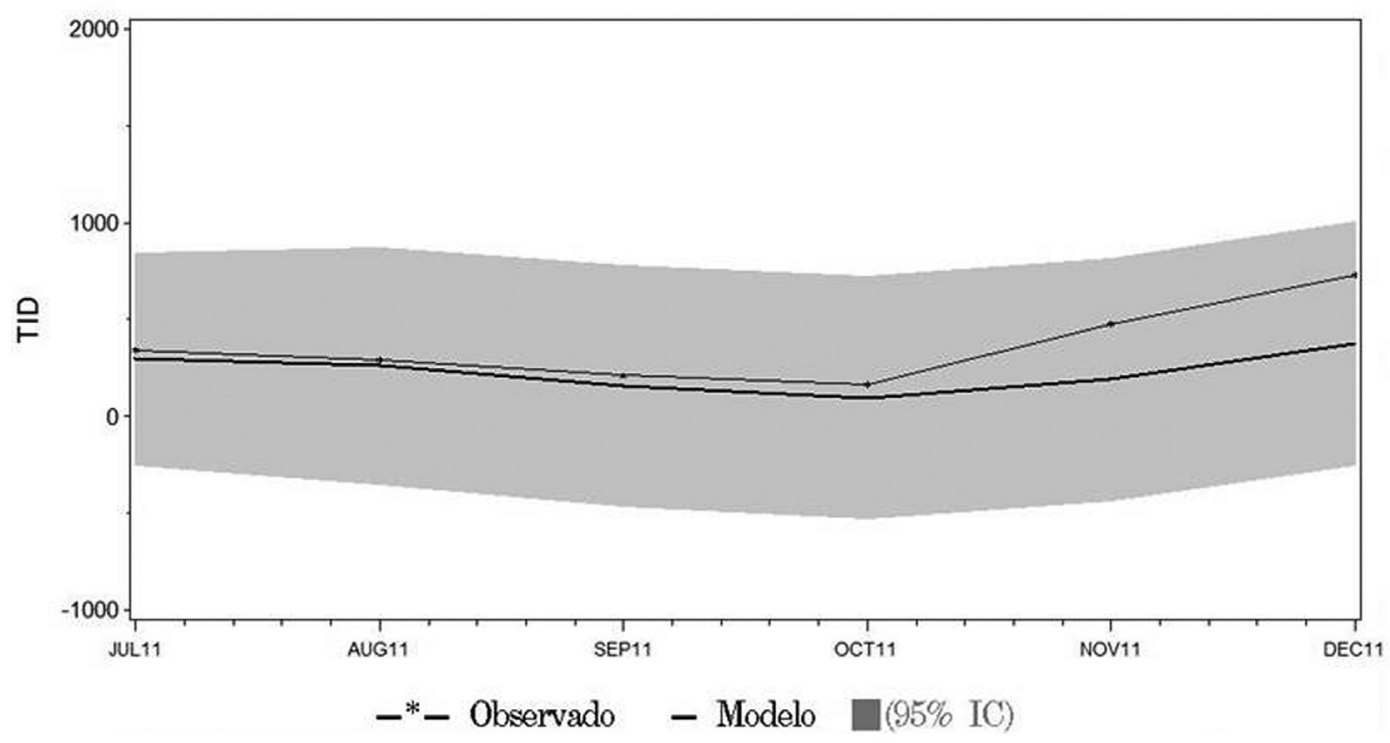

Figura 8 - Valores previstos pelo modelo multivariado e valores observados para a TID.

Tabela 1 - Previsões realizadas pelo modelo multivariado.

\begin{tabular}{lcccccc}
\hline Meses & TID (observada) & TID (prevista) & Casos de dengue & Precipitação & Erro 1 & Erro 2 \\
\hline Jul/11 & 343,66 & 296,41 & 168 & 195,3 & 0,1375 & 0,0189 \\
Ago/11 & 294,04 & 260,09 & 215 & 179,4 & 0,1155 & 0,0133 \\
Set/11 & 211,18 & 156,1 & 183 & 60,3 & 0,2608 & 0,0680 \\
Out/11 & 165,11 & 94,67 & 164 & 139,1 & 0,4266 & 0,1820 \\
Nov/11 & 474,88 & 188,8 & 352 & 174,6 & 0,6024 & 0,3629 \\
Dez/11 & 731,04 & 374,7 & 355 & 201,7 & 0,4874 & 0,2376 \\
& & & & & MAPE $=0,3383$ & RMSE $=0,3835$ \\
\hline
\end{tabular}

série mais longa, os intervalos de confiança seriam mais estreitos e, consequentemente, haveria um aumento na confiabilidade quando na comparação entre valores observados e previstos. A tendência, neste caso, seria que os valores previstos ficassem mais próximos dos valores observados.

A correlação entre as previsões da Taxa de Incidência de Dengue (TID) e valores observados ficou em 0,7904 com $\mathrm{p}<0,061$. Ou seja, para um nivel de significância menor que $10 \%$ pode-se considerar que as previsões e valores observados da Taxa de Incidência de Dengue (TID) são correlacionados.

O gráfico da Fig. 9 apresenta as médias com os erros-padrão das previsões e valores observados das Taxas de Incidência de Dengue (TID) com os respectivos errospadrão para o periodo de julho à dezembro de 2011. Observa-se que, em média, as previsões das Taxa de Incidência de Dengue (TID) foram menores que as observadas. $\mathrm{O}$ teste $t$ foi utilizado para a comparação entre as médias e apre- sentou como resultado um valor $t=-1,5009$ com $p$-value $=0,1643$. Neste caso, pode-se concluir que as diferenças entre as médias das previsões e valores observados da Taxa de Incidência de Dengue (TID) não são significativas. Pode-se, portanto, considerar que o modelo apresenta resultados satisfatórios e que pode ser utilizado em previsões de incidencia da dengue.

Os resultados deste estudo evidenciaram uma relação entre o padrão do regime de chuvas em cada espaço urbano e a Taxa de Incidência de Dengue (TID), ou seja, a sazonalidade bem demarcada desta doença não é causada por processos aleatórios. Este resultado está em consonância com outras investigações realizadas no Brasil e em outros países, ao considerar o componente chuva (Wu et al., 2007; Hurtado et al., 2007).

Nos anos considerados na pesquisa, a dengue ocorreu na área urbana de Belém em todos os meses do ano, com maior evidência nos meses considerados chuvosos, demonstrando que as condições climáticas são favoráveis 


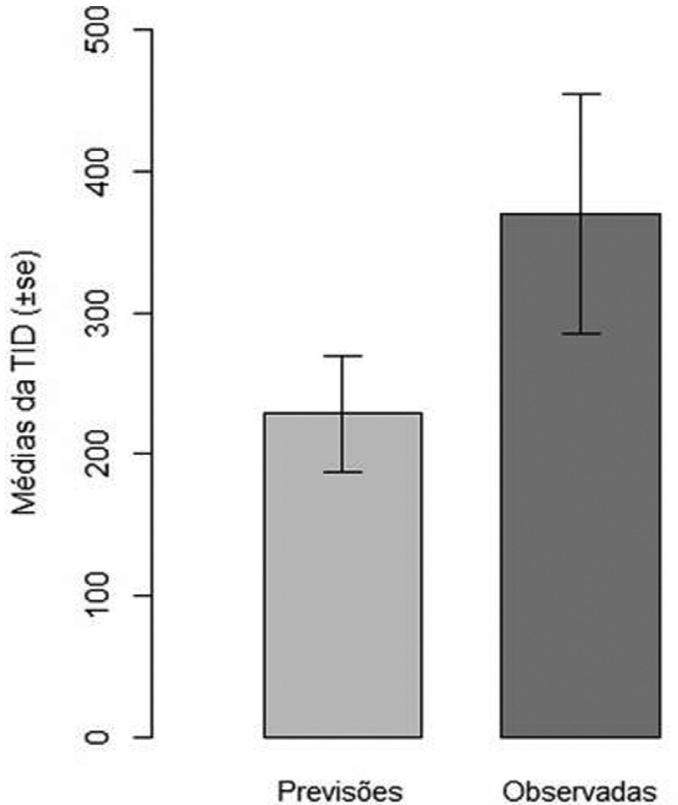

Figura 9 - Médias das previsões dos valores observados da TID com os respectivos erros-padrão.

para o desenvolvimento do vetor. Portanto foi possível verificar que a incidência da dengue na área urbana de Belém é maior nos meses de março, principalmente nos bairros do Marco, Guamá e Pedreira. Na área em estudo, Belém, se observou que o aumento da precipitação influencia no aumento da incidência de dengue no município.

Corroboram com os resultados deste estudo, o estudo feito por Gonçalves Neto e Rebêlo (2004) que mostrou que as chuvas não só aumentaram consideravelmente a quantidade de criadouros disponíveis para o desenvolvimento das formas imaturas do vetor, como também gerou condições ambientais mais apropriadas para o desenvolvimento de adultos.

Os modelos univariados utilizados para a incidência de dengue e precipitação foram utilizados e para a construção do modelo multivariado para a dengue, que apresentou resultados satisfatórios e, neste caso, pode ser utilizado na previsão de casos de dengue para área urbana de Belém. Esses resultados confirmaram o estudo feito por Pacheco (2001), em que os métodos utilizados para a análise foram os modelos de séries temporais clássicos de Box e Jenkins, em que as variáveis meteorológicas, umidade do ar, temperatura do ar e precipitação, foram estudadas através da função de correlação cruzada.

\section{Conclusões}

As análises dos dados de ocorrência de casos de dengue, representados pela Taxa de Incidência de Dengue (TID) e precipitação mostrou uma relação direta entre essas duas variáveis para o período de 2007 à 2011, observada claramente no período chuvoso (dezembro à maio).
Alguns estudos procuram demonstrar que o comportamento temporal da incidência de dengue considera condições ambientais e ainda sociais da população em geral. No entanto neste estudo tem-se incidência em bairros onde a população residente possui um padrão econômico e social diferenciados como é caso dos bairros do Marco e Pedreira, onde em um mesmo bairro há um paradoxo entre boa infraestrutura e a falta de saneamento básico.

$\mathrm{Na}$ etapa de validação do modelo, as previsões obtidas apresentou diferenças não significativas em relação aos dados reais, não incluso no modelo e, portanto, com potencialidade de ser utilizado em estudos de previsões.

Embora a série disponível não tivesse um tamanho ideal, em torno de 120 observações, a validação do modelo mostrou-se satisfatória, isto é, as previsões obtidas pelo modelo apresentou diferenças não significativas em relação aos dados reais e, portanto, com potencialidade de ser utilizado em estudos de previsões. Com um monitoramento adequado dos casos de dengue por parte dos órgãos públicos, espera-se, no futuro, dispor de séries mais longas e possivelmente com resultados mais precisos quando do uso da metodologia proposta.

\section{Agradecimentos}

Os autores agradecem à Secretaria Municipal de Saúde (SESMA), mais especificamente ao Departamento de Vigilância e Saúde (DEVS) pelos casos mensais de dengue notificados, ao Instituto Nacional de Meteorologia (INMET) pelos dados de precipitação e a Coordenação de Aperfeiçoamento de Pessoal de Nível Superior (CAPES) pela bolsa de mestrado concedida.

\section{Referências}

BARRETO, M.L.;TEIXEIRA, M.G. Dengue no Brasil: situação epidemiológica e contribuições para uma agenda de pesquisa. Estudos Avançados, v. 22, n. 64, p. 53-72, 2008.

BELÉM. Secretaria Municipal de Coordenação Geral do Planejamento e Gestão. Anuário estatístico do município de Belém. Belém, v. 17, p. 456, 2011.

CAUSEY, O.R.; THEILER, M. Virus antibody survey on sera of residents of the Amazon valley in Brazil. The American Journal of Tropical Medicine and Hygiene, v. 7, n. 1, p. 36-41, 1958.

COSANPA. Plano Diretor de Sistema de Esgoto Sanitário da Região Metropolitana de Belém, Manual Descritivo, v. 1, 1987.

CHOUDHURY, M.; BANU, S.; ISLAM, M.A. Forescasting dengue incidence in Dhaka, Bangladesh: A time series analysis. Dengue Bulletin, v. 32, p. 29-37, 2008.

EARNEST, A.; TAN, S.B.; WILDER-SMITH, A.; MACHIN, D. Comparing statical models to predict dengue fever notifications. Computational and Mathematical Methods in Medicine. 2012.

GASPARRINI, A.; ARMSTRONG, B. Time series analysis on the health ef- fects of temperature: Advancements and limitations. Environmental Research, v. 110, n. 6, p. 633$638,2010$. 
GHARBI, M.; QUENEL, P.; GUSTAVE, J.; CASSADOU, S.; RUCHE, G.L; GIRDARY, L.; MARRAMA, L. Time series analysis of dengue incidence in Guadaloupe, French West Indies: Forecasting models using climate variables as predictors. BMC Infections Diseases, v. 11, p. 166, 2011.

GONÇALVES NETO, V.S.; REBÊLO, J.M.M. Aspectos epidemiológicos do dengue no município de São Luis, Maranhão, Brasil, 1997-2002. Cad. Saúde Pública, v. 20, n. 5, p. 1424-1431, 2004.

HURTADO-DÍAZ, M.; RIOJAS-RODRÍGUEZ, H.; ROTHENBERG, S.J.; GOMEZ-DANTÉS, H.; CIFUENTES, E. Short communication: impact of climate variability on the incidence of dengue in Mexico. Trop Med Int Health, v. 12, n. 11, p. 1327-37, 2007.

INSTITUTO BRASILEIRO DE GEOGRAFIA E ESTATÍSTICA - IBGE. Disponível em: http://www.ibge.gov.br/censo2010/primeiros_dados_divulgados/index.php?uf $=15$. Acesso em 11 de jan. de 2012.

KOURI, G.; GUZMÁN, M.G.; VALDÉS, L.; CARBONEL, L.; DEL ROSARIO, D.; VAZQUEZ, S.; LAFERTÉ, J.; DELGADO, J.; CABRERA M.V. Remergence of dengue in Cuba: a 1997 epidemic in Santiago de Cuba. Emerging Infectious Diseases, Atlanta, US, v. 4, n. 1, p. 89-92, 1998.

LIANG, L.; HUALIANG, L.; LINWEI, T.; WEIZHONG, Y.; JIMIN, S.; QIYONG, L. Time series analysis of dengue fever and weather in Guangzhou, China. BMC Public Health, v. 9, p. 395, 2009.

LUZ, P.M.; MENDES, B.V.; CODEÇO, C.T.; STRUCHINER, C.J.; GALVANI, A.P et al. Time series analysis of dengue incidence in Rio de Janeiro, Brazil. Am J Trop Med Hyg, v. 79, n. 6, p. 933-939, 2008.

MORETTIN, P. A. Econometria financeira: um curso em séries temporais financeiras. $2^{\text {a }}$ edição. São Paulo: Edgar Blucher, 2011.

NAING, C.M; LERTMAHARIT, S.; NAING, K.S. Time-Series analysis of dengue fever/dengue haemorrhagic fever in Myanmar since 1991. Dengue Bulletin, v. 26, p. 24-32. 2002.

NATAL, D. Bioecologia do Aedes aegypti. Biológico. Ref Type: Journal, v. 64, n. 2, p. 205-207.2002.

HII,Y.L.; ROCKLOV, J.; NG. N.; TANG, C.S.; PANG, F.Y.; SAUERBORN, R.; Climate variability and increase in intensity and magnitude of dengue incidence in Singapore. Global Health Action, v. 2, n. 6, p. 1-9, 2009.

HURTADO-DÍAZ, M.; RIOJAS-RODRÍGUEZ, H.; ROTHENBERG, S.J.; GOMEZ-DANTÉS, H.; CIFUENTES, E. Short communication: impact of climate variability on the incidence of dengue in Mexico. Trop Med Int Health., v. 12, n. 11, p. 1327-1337, 2007.

FREITAS, M.G. R.; SCHREIBER, K.V.; TSOURIS, P.; WEIMANN, E.T.S.; MOURA, J.F. L. Associations between dengue and combinations of weather factors in a city in the Brazilian Amazon. Revista Panamericana de Salud Pública, v. 20, n. 4, p. 256-267, 2006.

PACHECO, F.G.A. Estudo da influência de variáveis meteorológicas no aparecimento de casos graves de leptospirose em Salvador - BA via modelos de séries temporais. Dissertação (Mestrado em Saúde Pública) - Fundação Oswaldo Cruz, Escola Nacional de Saúde Pública. 2001.
PINHEIRO, F.P. Los programas de erradicacion y de control Del Aedes aegypti em lãs Américas. OPS/HCP/HCT, 1996.

PROMPROU, S.; JAROENSUTASINEE, M.; JAROENSUTASINEE, K.; Forecasting dengue hemorrhagic fever cases in Southern Thailand using ARIMA Models. Dengue Bulletin, v. 30, p. 99-106, 2006.

REBELO, J.M.M.; COSTA, J.M.L.; SILVA, F.S.; PEREIRA, Y.N.O.; SILVA, J.M. et al. Distribuição de Aedes aegypti no Estado do Maranhão, Brasil. Cadernos de Saúde Pública, v. 15, n. 3, p. 477-486, 1999.

RIBEIRO, A.F.; MARQUES, G.; VOLTOLINI, J.C.; CONDINO, M.L.F. Associação entre incidência de dengue e variáveis climáticas. Rev. Saúde Pública, v. 40, n. 4, p. 671-676, 2006.

SANTOS, O.O. Estudo epidemiológico da dengue em Maceió, Alagoas, no período de 1997 a 2002. Tese (Doutorado em Saúde Pública)- Faculdade de Saúde Pública, Universidade de São Paulo, São Paulo, 2003.

SILAWAN, T.; SINGHASIVANON, P.; KAEWKUNGWAL, J.; NIMMANITYA, S.; SUWONKERD, W. et al. Temporal patterns and forecast of dengue infection in Notheastern Thailand. Southeast Asian. J Trop Med Public Health, v. 39, n. 1, p. 90-98, 2008.

SILVA, F.D.S.; COELHO, M.S.; LUCIO, O.S.; REBELLO, E.; RAMALHO, W.M. Caracterização Espacial da Incidência Sazonal da Dengue no Brasil. In: CONGRESSO BRASILEIRO DE METEOROLOGIA, 14. Florianópolis. Anais... Florianópolis: Congresso Brasileiro de Meteorologia, 2006.

SOEBIYANTO, R. P.; ADIMI, F.; KIANG, R.K. Modeling and predicting seasonal influenza transmission in warm regions using climatological parameters. Plos One, v. 5, n. 3, p. 110, 2010.

SOUZA, S.R. Fatores associados à ocorrência de formas imaturas de Aedes aegypti na Ilha do Governador, Rio de janeiro, Brasil. Revista da Sociedade Brasileira de Medicina Tropical, v. 32, n. 4, p. 373-382, 1999.

SUDAM. Atlas climatológico da Amazônia brasileira: Projeto hidrologia e climatologia da Amazônia. Belém, v. 1, n. 39, p. 125, 1984.

TEIXEIRA, M.G.; COSTA, M.C.N.; BARRETO, M.L.; MOTA, E. Dengue and dengue hemorrhagic fever epidemics in Brazil: what research is needed based on trends, surveillance, and control experiences? Caderno de Saúde Pública, Rio de Janeiro, v. 21, n. 5, p. 1307-1315, 2005.

TIAN, L.; BI, Y.; HO, S.C.; LIU, W.; LIANG, S.; GOGGINS, W.B.; CHAN, E.Y; ZHOU, S.; SUNG, J.J. One-year delayed effect of fog on malaria transmission: a time-series analysis in the rain forest area of Mengla County, southwebst China. Malaria Journal, v. 19, n. 7, p. 110, 2008.

WEI, W.S. Time Series Analysis: Univariate and Mutivariate Methods. Addison-Wesley Publishing Company, 1990.

WILDER-SMITH, A.; EARNEST, A.; TAN, S.B.; OOI E.E.; GUBLER, D.J. Lack of association of dengue activity with haze. Epidemiol Infect, v. 138, n. 7, p. 962-967, 2010.

WONGKOON, S.; POLLAR, M.; JAROENSUTASINEE, M.; JAROENSUTASINEE, K. Predicting DHF incidence in Northerm Thailand using time series analysis technique. Proc Wordl Acad of Sci Eng Technol, v. 1, n. 8, p. 484488, 2007. 
WONGKOON, S.; JAROENSUTASINEE, M.; JAROENSUTASINEE, K. Climatic variability and dengue virus transmission in Chiang Rai, Thailand. Biomedica, v. 27, n. 3, p. 5$13,2011$.

WONGKOON, S.; JAROENSUTASINEE, M.; JAROENSUTASINEE, K. Development of temporal modeling for prediction of dengue infection in Northeastern Thailand. Asian
Pacific Journal of Tropical Medicine, v. 5, n. 3, p. 249$252,2012$.

WU, P.C.; GUO, H.R; LUNG, S.C.; LIN, C.Y.; S.U, H.J. Weather as an effective predictor for occurrence of dengue fever in Taiwan. Acta Tropica, v.103, n. 1, p. 50-57, 2007.

This is an Open Access article distributed under the terms of the Creative Commons Attribution Non-Commercial License which permits unrestricted non-commercial use, distribution, and reproduction in any medium provided the original work is properly cited. 